\title{
Resonancias del zapatismo mexicano y la resistencia palestina: dos ejemplos de autonomía en el Sur Global
}

\author{
Resonances of Mexican Zapatismo and \\ the Palestinian resistance: two examples \\ of autonomy in the Global South
}

doi: http://dx.doi.org/10.32870/

espiral.v23i65.4457

Moisés Garduño García

\section{Resumen}

Este artículo muestra algunas de las resonancias existentes entre la resistencia zapatista y la resistencia palestina como dos ejemplos de glocalidad. El objetivo es establecer algunas zonas de contacto que puedan recrear un ejemplo de traducción cultural en el denominado Sur Global, atendiendo los obstáculos que cada una de estas luchas enfrenta respectivamente. Se concluye que de Chiapas a Nablus existen muchos elementos que la gente comparte cuando está en un proceso de resistencia, y se hace especial énfasis en las actividades glocales, definidas como aquellas acciones locales con un impacto global, las cuales incluyen la construcción de escuelas, clínicas y otros espacios de autonomía local que en su andamiaje sirven para deconstruir ideologías y prácticas dominantes que homogenizan a los pueblos en nombre de la globalización neoliberal.

Palabras clave: tierra, dignidad, autonomía, glocalidad, globalización.

\begin{abstract}
This article shows some of the existing resonances between the Zapatista and the Palestinian resistances as examples of glocalization. The objective is to establish some contact zones that can recreate an example of cultural translation in what is known as the Global South, addressing the challenges that each of these struggles faces respectively. It concludes that from Chiapas to Nablus there are several elements shared by people when they are in a process of resistance; emphasis is placed on the glocal activities, defined as local actions with a global impact. These activities include the construction of schools, clinics, and other spaces of local autonomy, whose structures serve to deconstruct ideologies and dominant practices that homogenize the people in the name of neo-liberal globalization.
\end{abstract}

Keywords: Land, dignity, autonomy, glocalization, globalization.

\footnotetext{
- Profesor-Investigador de la Facultad de Ciencias Políticas y Sociales, UNAM. mgarduno@politicas.unam.mx
} 
Introducción'

Ante el debilitamiento de la capacidad redistributiva del Estado, su destacado papel en la privatización de la tierra, el aumento de la vigilancia, el castigo de sus propios ciudadanos y la crisis de representación asociada con el uso sistemático de la violencia, muchos pueblos marginados han recurrido a organizaciones no gubernamentales (ONG) y a organismos jurídicos internacionales con la esperanza de equilibrar las desigualdades y reclamar los derechos que les han sido negados. Sin embargo, la persistencia de condiciones de pobreza y de violencia ha dado pie a otro tipo de búsqueda en el cual las autonomías locales, como narrativas de desobediencia epistémica a nivel internacional, han planteado retos significativos al sistema que las margina. ${ }^{2}$

Algunas de esas respuestas son resonantes entre sí, dada la similitud del tipo de problemas a los que se enfrentan y la sistematización de las violencias (física, epistémica, económica y psicológica) a las que han sido expuestas, y a las cuales contestan. A pesar de que cada escenario de

I. Este artículo fue posible gracias a la retroalimentación lograda en la conferencia internacional "Living, Consuming and Action in Glocal Palestine", organizada por la Universidad de An Najah, de Nablus, Palestina, y el French Institute of the Near East, en colaboración con el French Institute of Jerusalem, en la cual participé entre los días 30 de septiembre y 2 de octubre de 2014 con el apoyo de la Agencia Mexicana para la Cooperación Internacional y el Desarrollo (AMEXCID), la Facultad de Ciencias Políticas y Sociales de la UNAM y el Centro de Investigaciones y Estudios Superiores en Antropología Social (CIESAS), sede Occidente.

2. Las autonomías locales han sido definidas por investigadores como Jorge Alonso Sánchez como uno de los mejores ejemplos de la cotidianidad de la democracia participativa. Incluso, más allá de esta definición de democracia, el mismo autor ha ahondado para hablar del "gobierno de los libres", donde las autoridades de diversas localidades han resignificado la dimensión de "gobierno" para dotarle de un sentido de responsabilidad y a partir de ello dignificar la libertad de decidir por sí mismas. Alonso ha acuñado el término demoeleuthería para referirse a este fenómeno, siendo las practicas locales del zapatismo la base principal de su teoría. En este sentido,"lo local" se relaciona con la forma en la que las comunidades deciden por sí mismas sus formas de gobierno, sus formas de producción, distribución y consumo de sus bienes, su cultura y su pensamiento (véase Alonso, 2015). 
resistencia es diferente, hay casos que invitan a ser abordados en paralelo debido a que lo que tienen en común en cuanto a sus articulaciones y sus experiencias de traducción cultural sirve como ejemplo para desmantelar supuestos teóricos que mantienen a la resignación y al sometimiento al capitalismo y la globalización neoliberal como únicos caminos hacia el progreso y el supuesto desarrollo humano (Huntington, 1993, p. 15). ${ }^{3}$

En este contexto es que este artículo se propone abordar las resistencias palestinas y zapatistas como dos proyectos contra esta globalización neoliberal que presentan diversos mecanismos, respuestas y herramientas para superar los limitados modos de movilidad, territorialidad, producción, consumo, salud y economía a los que han sido expuestos por el propio "aperturismo globalizador", el cual, en el caso de Palestina, es representado por la economía de ocupación militar llevada a cabo por Israel, y en el caso zapatista, por el hostigamiento paramilitar del Estado mexicano y el tipo de economía extractivista que este fomenta, tomando en cuenta que ambos escenarios son parte del mismo proceso de lo que los zapatistas acuñaron como "la cuarta guerra mundial o la guerra contra la humanidad" (subcomandante insurgente Marcos, 1999), la cual consiste en la destrucción de los territorios y su despoblación para, posteriormente, reconstruir, reordenar y marcar un ritmo económico para la recuperación del capitalismo.

Las experiencias zapatistas y palestinas deben ser enmarcadas en el contexto actual de una geografía de la destrucción y de historicidio, es decir, de un momento en el cual la historia y la geografía pretenden ser secuestradas

3. Si bien la globalización fue presentada por algunos intelectuales cercanos a Huntington como Raz (1986) o Fukuyama (1992) como un proceso que coadyuvaría al desvanecimiento de las fronteras nacionales en aras de responder a los problemas de la sociedad, algunos pueblos han experimentado los efectos más devastadores de este discurso liberal al verse desposeídos y marginados (Vivas y Forcades, 2013, pp.I2-15). 
y distorsionadas para beneficio de ciertas redes de poder en que algunos actores se encargan de marginar o excluir, mediante su corrupción, asesinato o desaparición sistemática y premeditada, a aquellos pueblos que no encajan en sus proyectos estructurales. Palestinos y zapatistas se han enfrentado, en diversas formas y contextos, a criterios de inclusión y exclusión estatal, de violencia, acoso policial y sexual, enfrentamientos contra el Ejército, luchas contra empresas transnacionales, infiltrados, grupos paramilitares, agencias de inteligencia, bloqueos económicos, asesinatos selectivos, provocaciones, corrupción de ciudadanos, despojo de tierras, intentos de limpieza étnica, racismo, discriminación y otros obstáculos para la solución de sus demandas, que tienen que ver con la autonomía y una serie de derechos para los pueblos indígenas, en el caso zapatista, y con independencia en el caso palestino.

La historia moderna de los países donde ambos grupos poblacionales llevan a cabo sus luchas está marcada por una serie de pérdidas territoriales desde finales del siglo XIX, en las cuales la táctica de movilizar familias enteras, campesinos y trabajadores, bajo una retórica de "la búsqueda de la prosperidad", era un componente importante de las lógicas de la dominación imperial y de sus agentes en el mundo, principalmente entre los lobbies sionistas de la Inglaterra de aquella época y los políticos estadounidenses ideólogos de la doctrina del destino manifiesto (Brinley, 1951, p. 11).

Hoy, frente a mantas y eslóganes que muestran frases como "1948 es igual a 1492", se sabe que la colonización, la modernización y el militarismo aplicados en Chiapas y en Palestina han creado escenarios de violencia estatal y asesinatos masivos como el de Acteal, en 1997, y el de Sabra y Shatila, en 1982, respectivamente, en los cuales los problemas de la pérdida de territorio, el despojo y la intimidación han sido una experiencia común y amarga para ambos casos. 
Actualmente, autores como Johnson y Quiquivix (2013), con base en algunos informes desclasificados del Departamento de Defensa de los Estados Unidos, ${ }^{4}$ han documentado "los esfuerzos de cooperación entre México e Israel para tener la tecnología armamentista suficiente que será usada contra movimientos de insurrección" (Milenio, 2013), donde, en el caso mexicano, el Ejército Zapatista de Liberación Nacional (EZLN) ha sido un objetivo claro, al menos desde 1994; en el caso palestino, la propia población, algunos activistas y líderes de organizaciones militares como $\mathrm{Al}$ Fatah, lo han sido a su vez prácticamente desde 1948 (y otros, como Hamas, desde 1987) (Pappé, 2006).

Dicha cooperación se ha basado, sobre todo, en la "coordinación de la seguridad en el ámbito de la policía, las prisiones y el uso eficaz de la tecnología orientada a compartir experiencias, en el combate al terrorismo" (Johnson y Quiquivix, 2013, p. 8), acciones que son parte de una larga historia de cooperación entre ambos países en estos temas. ${ }^{5}$

Además, palestinos y zapatistas han experimentado la destrucción de su medio ambiente a través de la deforestación, la confiscación y la contaminación de tierras, fenómenos que han servido como herramienta de control de las vidas y los medios de vida de dichos pueblos, tal como ejemplifica el caso de Deir Sharaf, cerca de Nablus, donde empresas israelíes han vertido sus residuos sólidos y peligrosos en una antigua cantera, sin preparación y sin regulación, de la misma forma que diversas empresas trans-

4. Parte de los documentos mencionados fueron recuperados de: http://www2. gwu.edu/ nsarchiv/NSAEBB/NSAEBB I09/9405 I I.pdf

5. México comenzó a recibir armamento israelí en 1973 por parte de la Israel Aerospace Industries. Entre 1970 y 1980, las exportaciones continuaron en forma de armas pequeñas y vallas electrónicas, aumentando entre 2003 y 2004, cuando México compró helicópteros, drones y otorgó licitaciones a empresas israelíes para entrenar personal de la policía federal. Entre las empresas israelíes que recibieron contratos por parte de Gobierno mexicano están Magal Security Systems, Elbit Systems y Verint Systems. 
portistas lo han hecho en el municipio de Reforma, Chiapas, y en otros estados de la república mexicana.

Otro factor más que justifica el estudio de estas resistencias en el contexto de la geografía de la destrucción ha sido el establecimiento, distorsión y destrucción de los censos de población que, en el caso de Israel, en 2006 fueron destruidos junto con los nombres de civiles candidatos para la gobernación de Nablus, Palestina (Abu-Hiljah, 2009); en el caso mexicano, dichos instrumentos ignoraron a sesenta pueblos indígenas con sus lenguas y culturas cuando se realizó el Censo Nacional de Población del año 2012 (Aboites, 2013). Lo mismo ha ocurrido con la historia indígena en ambas luchas, donde los libros de texto de educación básica del Gobierno mexicano, y los textos homólogos editados por el Gobierno israelí, han borrado gradualmente la historia, lengua y cultura maya y palestina, respectivamente, junto con sus manifestaciones culturales, significados y formas de crear y construir el pensamiento (Peled, 2012; Pacheco, et al., 2011).

Con lo anterior, ¿cómo se puede concebir o diseñar el desarrollo y el futuro de una sociedad en un aparente mundo globalizado donde palestinos y zapatistas, y muchos otros pueblos en el mundo, están experimentando un encarecimiento de la movilidad (elemento que supuestamente es crucial para la comprensión de un mundo globalizado), ${ }^{6} \mathrm{y}$ un hostigamiento militar y paramilitar, asesinatos selectivos y otros factores que son perpetrados en nombre de la seguridad nacional del Estado? Ciertamente, la resistencia palestina tiene grandes diferencias con el movimiento zapatista en sus objetivos políticos, sus actores y sus tiempos.

\footnotetext{
6. Hay varios conceptos de movilidad. En este texto se entiende por movilidad a la condición física y administrativa que permite y facilita el acceso de las personas al trabajo, la escuela y a otros servicios necesarios para tener una vida digna. La movilidad se entiende así como esa libertad de acceso a los bienes y servicios, a través de la espacialidad, que ofrece el Estado.
} 
La consecución de un Estado, por ejemplo, ha predominado en el lenguaje contestatario palestino desde el inicio de la $n a k b a,{ }^{7}$ mientras que en el zapatismo un Estado independiente no es necesario en la formulación del "mandar obedeciendo" ni en el espíritu de las asambleas, que son el corazón que determina las decisiones que acata la comunidad zapatista.

No obstante, una consideración importante, como campo de resonancia, es el estudio de la cotidianeidad de las luchas y no sólo de los rostros que las hacen públicas. Esto es, la atención que se debe a la idea de que los zapatistas no son (solamente) lo que comunicaba el subcomandante Marcos (hoy Galeano), o que los palestinos no se determinaban (solamente) por las decisiones que tomaba Yasser Arafat o cualquier líder de Hamas, o incluso la búsqueda de que ninguno de estos pueblos sea determinado, estudiado o analizado por el nivel de opresión que experimenta en diferentes niveles y formas, invitando a mirar hacia las prácticas autónomas y cotidianas como un pilar fundamental para el entendimiento de las bases sociales y sus utopías.

Así, es a través de los colectivos que están al margen de dichas esferas políticas, y que adquieren estrategias muy diferentes para expresar su indignación, que se puede encontrar un campo resonante entre las resistencias de carácter amplio y dialógico. Con dichas prácticas se puede hacer un ejercicio de espejos y dilucidar que, tal como diversos mensajes zapatistas y palestinos lo han confirmado (Rohana, 2014; EZLN, 2009), se trata, ante todo, de luchas fraternales que operan con y a través de una fuerte cultura de autonomía en que la misma autogestión no es un fin o el punto culminante de toda resistencia popular, sino que se

7. En lengua árabe, nakba quiere decir "catástrofe", y se refiere al acontecimiento que marcó el inicio del conflicto con Israel en 1948. Entonces, mientras para Israel 1948 es el año de la independencia, para Palestina es el año de la catástrofe. 
le piensa primordialmente como una estrategia o un medio sin el cual la propia resistencia existiría más difícilmente.

\section{Autonomía y autogestión como pilares \\ de la glocalidad: consideraciones teóricas \\ para el estudio de las resistencias}

El término glocal puede ser entendido como aquella serie de acciones locales y otras prácticas de resistencia y autogestión al interior de una comunidad que, con su materialidad y diseño, alternativo a la tendencia homogenizadora de la globalización, rompen con los modelos neoliberales de producción, distribución y consumo, y consiguen un impacto global para diversos sujetos que lo conocen, lo propagan o lo acompañan en su cotidianidad.

Estas acciones en sí no se proyectan como un paradigma discursivo o epistemológico, sino simplemente como un ejemplo (entre muchos más) de que las cosas se pueden hacer de manera alternativa a como las maneja y diseña el Estado. Las actividades glocales, en este sentido, ayudan a descentralizar el concepto de "desarrollo" de los marcos "estatocentristas" de referencia, y permiten vislumbrar otras formas de vida digna donde se resiste, y en muchos casos se sobrevive, a las políticas de marginación producidas por el capitalismo global.

Se trata, en corto, de potenciar las prácticas autónomas como evidencias de una reproducción diferente del espacio y el tiempo, en la cual se ve al territorio no como una demarcación del Estado y de su poder sobre los ciudadanos, sino como una relación de pertenencia y dependencia de los sujetos con esa tierra a partir de la cual se pueda vivir libremente y con dignidad. Esto, automáticamente, representa una amenaza al poder, la demarcación, y la territorialización clásica y geopolítica del Estado, el cual estará en contra de dichos proyectos intentando minarlos, criminalizarlos o 
aterrorizarlos mediante diversas técnicas disuasivas o de acción directa.

La glocalidad se convierte así en una utopía presente, es decir, en una serie de ejemplos que enseña al mundo cómo se está resistiendo la opresión del Estado y las multinacionales con base en el trabajo de la propia tierra, la autoorganización de los recursos y la redistribución de los productos e ideas en bienestar del colectivo (esto como contrapeso al individualismo pregonado por el neoliberalismo), sin tener que esperar a que los actores que encabezan el modelo capitalista desaparezcan o caigan en crisis. Se trata de una resignificación del trabajo, del consumo y del espacio que permite generar experiencias diferentes de espacialidad ante un contexto que asfixia porque, por un lado, la agricultura artesanal está bajo intensa presión por la confiscación de tierras por parte del Estado y, por otro, el mercado está saturado de productos provenientes de empresas transnacionales que a menudo cultivan en tierras confiscadas ilegalmente a los pueblos.

Ante esto, las opciones para la resistencia pueden parecer bastante limitadas, pero en los últimos veinte años las experiencias de espacialidad en Chiapas y en Palestina se han definido por la manera en que una comunidad global de millones de personas se mantiene aparentemente bien comunicada con lo que pasa alrededor de poblaciones fragmentadas, ya sea por los asentamientos, la marginación o la deshumanización de los puestos militares y paramilitares que acosan a palestinos y zapatitas.

Este fenómeno mediático que conecta la vida local con lo que se habla de ella a escala global también se ha traducido con el término glocal (en árabe mauhal), es decir, un concepto que permite describir la forma en la que el denominado proceso de globalización económica ha sido experimentado y reapropiado por pueblos cuyos mundos físicos se han vuelto, paradójicamente, unos de los más 
restringidos, militarizados, acosados y vigilados del planeta. El hecho de que las practicas locales trasciendan internacionalmente, se hable de ellas, en algunas ocasiones sean emuladas, se compartan, se critiquen o complementen es precisamente lo que las hace valiosas y fuertes en sus comunidades respectivas, sobre todo porque se enfrentan a un mismo sistema neoliberal que es cuestionado y contestado de diversas formas y en varios continentes.

En este sentido, como concepto, la glocalidad es una noción que permite crear medios alternativos de producción de conocimientos a partir de las prácticas locales y la autogestión de las comunidades organizadas, con las cuales se pueden hacer diagnósticos diferentes de la realidad inmediata y, al menos para algunos teóricos, incluso de la realidad global y de sus problemas, a la vez que se pueden formular soluciones alternativas a las propuestas hechas por los Estados y otras agrupaciones como los partidos políticos o las ONG (González, 2003). La glocalidad también es una forma de crear utopías y nuevas formas de actuar en colectivo con diálogos y acciones que se convierten en experiencias sociales cercanas a lo que John Holloway (2011) denomina la "fragmentación del capitalismo", esto porque ocurre en diversas partes del mundo de manera simultánea, compleja e incluso rizomática. ${ }^{8}$

Manuel Castells (2012, pp. 72-73) se refiere a este fenómeno como espacios de contrapoder desde donde se crea

8. El rizoma es un concepto acuñado por Deleuze y Guatari (1972) que hace referencia a las multiplicidades en las que se presentan el cambio social y la organización de las colectividades. Se toma de la noción botánica del rizoma que, como planta que se extiende, ocupa espacios de terreno favorables, con lo cual se contrapone con la idea del árbol, el cual se enraíza profundamente en la tierra como un punto estático que crece tan sólo hacia arriba, sin abarcar ningún otro espacio a su alrededor. El rizoma, entonces, crece en diferentes direcciones simultáneamente, $y$ es usado como una metáfora para entender en las ciencias sociales y las humanidades la forma en la que un elemento social afecta a otro sin tener un centro de poder (líderes o rostros políticos), y que cambia en la medida que aumentan sus conexiones. 
la utopía que contesta la realidad impuesta por diversas redes a partir del despliegue de sujetos en resistencia. Santos (2014), en una carta a los jóvenes de México, llama a estas prácticas zonas liberadas, haciendo énfasis en que en estos espacios es donde los procesos de diálogo, las diversas concepciones de la educación, el libre pensamiento, la redefinición de ideas, la desesperanza y la esperanza, las decisiones tomadas en las asambleas, las asambleas mismas, la formación de comisiones o juntas de gobierno, las cooperativas, las coordinaciones, entre otras cuestiones, se materializan gracias a los mismos participantes indignados a través de programas de organización colectiva, tanto rural como urbana.

Pero para entender mejor las formas de reapropiación del espacio, de la tierra o el barrio a través de prácticas autónomas como baluartes de la glocalidad en varios escenarios de protesta en el mundo, es necesario considerar tres ideas importantes que se expondrán en el segundo apartado del presente artículo.

Una primera consideración es que la autogestión, como elemento de la glocalidad, varía según la causa, incluso de un colectivo a otro. Ciertas formas de acción autogestiva han supuesto algunas contradicciones en su andamiaje, cuestión que hay que aceptar y analizar sobre todo cuando se habla de que las zonas donde el zapatismo y los palestinos ejercen su autonomía no tienen las mismas características. Dicho de otro modo, no es lo mismo poseer tierra en la que se pueda cultivar, por muy precariamente que sea, la comida más básica para una familia, que vivir en un contexto urbano donde los edificios, la ocupación militar y otros factores como la demografía y la contaminación hacen que lo anterior no sea una opción viable (Holloway, 2005, p. 172). Algunos grupos urbanos autónomos palestinos, por ejemplo, han tenido que costear la ocupación a través del consumo de los productos israelíes que son cultivados en las tierras confiscadas y 
que son más baratos que los cultivados al interior de la tierra palestina. Aquí, la contradicción estriba en consumir productos israelíes para seguir resistiendo (Habibi, 2009). En el caso de los zapatistas, Holloway (2005, p. 177) pone como ejemplo el hecho que hayan tenido el gran mérito de reconocer desde un principio la contradicción de tener una organización militar en un movimiento que lucha por la dignidad humana y la horizontalidad de las relaciones sociales.

Por lo tanto, es menester decir que la autonomía no es un dogma, y que puede ser pensada y practicada desde muchos ángulos, urbanos, no urbanos o semiurbanos, donde las prácticas se componen de una mezcla de elementos regulares e irregulares de empleo, consumo y organización, porque es justo en medio de esas contradicciones, problemas y limitaciones, de su aceptación y canalización, donde se crean las condiciones necesarias para la supervivencia y el seguimiento de la resistencia y de la misma autonomía.

En este sentido, una segunda consideración es aquella que se refiere a la emergencia de una plétora de utopías que recrean la realidad en estos espacios autónomos y dotan de significado la práctica local, lo que facilita que se produzcan nuevos sujetos conscientes y críticos del capitalismo global. Es decir, en oposición a una ideología determinista que intentaría justificar el orden de las cosas tal como aparentan ser (Giraldo, 2013, pp. 7-10), la glocalidad implica hablar de una semiótica de la superación, la cual reinterprete no sólo el presente, sino el futuro a partir del presente, esto a pesar de la existencia de los simbolos culturales sobre los cuales se sustenta el sistema que se quiere superar. Un ejemplo claro que se detallará más adelante es la existencia del banco zapatista, una práctica que se resiste a la idea de la banca capitalista y la usa como herramienta de contrapoder en beneficio de la comunidad.

Otros ejemplos del acto como ideología, o mejor dicho, el acto como utopía, han sido aplicados en proyectos como los 
Caracoles y la Escuelita, en el caso zapatista, y en el caso de algunas prácticas del pueblo palestino como la cooperativa Sharaka o la Unión de Agricultores Palestinos, que a través de la materialización de proyectos como granjas para el autoconsumo, el Museo del Holocausto Palestino, o la campaña Boicot, Desinversiones y Sanciones (conocido en el mundo como BDS) han demostrado cómo se puede superar la opresión neoliberal y gestar otro tipo de relaciones sociales a través de propuestas y proyectos útiles no sólo para mantener la resistencia, sino necesarios para la misma subsistencia de las personas. En palabras de Zibechi (2014), se trata de "propuestas con acumulación de fuerzas en silencio y de la necesidad de esperar activamente a que el pueblo se decida a actuar sin la intención de tomar el poder" (p. 2).

Esto da pie a una tercera consideración, que aborda el peso de la palabra. Si bien en las autonomías se definen en gran medida el pensamiento de emancipación y la desobediencia epistémica a la globalización, la narrativa de estos actos es sumamente importante para dotarles de significado. Aquí interviene el uso y el valor de la palabra como motor de compromisos y lealtades con respecto al grupo. Ciertamente, en las comunidades zapatistas y palestinas muy pocas personas hablan sobre teorías sociológicas occidentales y rimbombantes porque las cosas, cuando se practican y funcionan, no causan la necesidad de teorizarlas, sino más bien de compartirlas para que sigan funcionando y de asentar los errores para que funcionen cada vez mejor.

En este sentido, la oralidad ha sido algo muy importante entre palestinos y zapatistas, ya que el peso y la importancia de la palabra son tales en ambas luchas que estos elementos acompañan la ley, la ética, el arte, la economía y el día a día como respuesta a las mentiras del opresor y sus redes. Un par de grandes ejemplos de cómo la palabra determina gran parte de los compromisos y los actos de las 
comunidades en ambos pueblos son la poesía y el muralismo, ambas muestras de identidad indígena y de reapropiación del espacio que sostiene la (re)producción de sus narrativas, y de su significado como pueblos en resistencia (Vargas, 2012; Eqeiq, 2013). ${ }^{9}$

Eso nos lleva a una cuarta consideración, que es la disciplina y la importancia del grupo en estos proyectos, entendiendo la disciplina no como aquella categoría que vigila, corrige, encierra y, en palabras de Foucault (1977), "adquiere una división binaria que controla las operaciones del cuerpo para garantizar la sujeción a una relación de docilidad-utilidad" (p. 124), sino entendida como una lealtad a las decisiones de las mayorías que se atiene a un bien colectivo.

La disciplina es la capacidad que, en lugar de decir qué es lo que se permite hacer o no, crea las condiciones donde las personas discuten y deciden sus asuntos, sin líderes y sin demandas "de arriba". La disciplina colectiva o comunitaria, el respeto por el otro y su dolor, o la preocupación por su felicidad son elementos que coadyuvan al proceso de subjetivación en tanto no hay expertos que recomienden qué hacer sino gente que aprende a buscar un camino para salir de los problemas con sus propias fuerzas, en un espíritu de grupo. En eventos como la manifestación de 2008 en $\mathrm{Na}^{\prime}$ alen, al oeste de la ciudad de Ramallah, o la toma simbólica de San Cristóbal de las Casas, Chiapas, por los zapatistas, en 2003, se ha podido observar una disciplina de grupo donde se dejan claras, por consenso, las formas

9. Existe un trabajo de la Dra. Amal Eqeiq sobre el diálogo de las literaturas indígenas, particularmente de la literatura contemporánea maya en Chiapas, México (1983-20I0) y la literatura palestina en Israel (1976-20I0). En su trabajo, la autora compara las tradiciones literarias de dos comunidades indígenas emergentes en el denominado Sur Global y defiende que el contexto histórico de la conquista española católica de Mesoamérica, en I519-1524, y el establecimiento de Israel, en 1948, como un Estado judío, crearon una ruptura en las historias y geografías mayas y palestinas que condujo a la marginación de estos pueblos.

\section{8}


de manifestación a realizar, las cuales son convenidas por consenso antes de las marchas o plantones. ${ }^{10}$

\section{De la violencia libertaria y la ley como técnica de dominación}

En este sentido, se tienen que problematizar dos factores presentes en algunos procesos de autonomía y resistencia con impacto global.

En primer lugar, se encuentran las respuestas autónomas no pacifistas, llamadas "de violencia libertaria" o "de autodefensa". Aunque la mayoría de las muestras de activismo urbano (sobre todo en grandes ciudades como El Cairo, Ciudad de México, Hong Kong o Estambul) han apostado por convenciones pacíficas de protesta, válidas y legítimas por sus actores, existen otros escenarios donde el pacifismo difícilmente ayuda a la resistencia o, incluso, a la misma supervivencia de la causa. Se puede enunciar, por ejemplo, el caso del surgimiento de grupos de autodefensa en Tepalcatepec, Michoacán, México, donde familias enteras han usado las armas como último recurso capaz de garantizar su vida ante el envolvente avance de organizaciones criminales en la zona (Rivera, 2014), o el caso de grupos de mujeres kurdas en zonas como Kirkuk, Iraq, o Kobane, Turquía, quienes han luchado contra grandes contingentes armados pertenecientes a la Organización del Estado Islámico (Daesh) para defender sus propiedades, sus vidas y las de sus hijos (Albayrak y Coker, 2014).

Problematizar sobre lo anterior implica una apertura epistémica capaz de entender las formas y contextos del uso

10. Eventos como la marcha de $\mathrm{Na}$ alen, que incluyen marchas en contra del muro israelí, son muy parecidos a la toma simbólica de edificios públicos que han realizado los zapatistas. En esos eventos, se deja claro qué se va a hacer, cómo se hará, cuánto tiempo durará, puntos de reunión, entre otras cuestiones de logística y disciplina. 
de la violencia en espacios autónomos, pues gran parte de ella se produce en territorios cuya lógica es la de la reproducción del espacio, en tanto garante de la vida, de las costumbres y de la cultura, y donde el despojo de tierras, la marginación y la represión diaria implican, como se ha mencionado, una lucha que, a pesar de haberse canalizado en un primer momento de manera institucional, diplomática, teórica o en una mesa de negociación, al final del día no está exenta de estar marcada por asesinatos, encarcelamientos y desapariciones de los representantes de esos grupos marginados (Tehutle, 2014).

En los medios de comunicación oficiales, por ejemplo, la condena del derecho a la autodefensa y a la utilización de la violencia como recurso de libertad emocional, política y cultural inunda los ojos de televidentes y lectores de prensa escrita. ${ }^{11}$ Parafraseando a Castellanos y Jiménez (2007, p. 225), este fenómeno termina siendo un sinsentido en tanto que aquel que condena los actos en su línea editorial no necesariamente vive la misma realidad y contexto de quien ejerce dicha acción; por tal motivo, aquella persona que señala la violencia sin entender el tejido social donde se produce irremediablemente correrá el riesgo de criminalizar la misma vida y el contexto que no conoce. Siguiendo a Todorov (2008), "no basta con condenar la violencia pues si queremos impedir que se vuelva a producir, es preciso intentar entenderla, ya que nunca estalla sin razón” (p. 140).

II. Hay que recordar que las manifestaciones palestinas y zapatistas siempre han estado relacionadas por los medios de comunicación oficiales con grupos criminales o terroristas. En el caso zapatista, se subrayó el supuesto nexo del EZLN con bandas de narcotraficantes e, incluso, durante el gobierno de Ernesto Zedillo se les acusó de terrorismo (Rico, 1995). En el caso palestino, se recuerda cómo cada manifestación ha sido presentada como un acto de terrorismo, o cuando se relacionó a Hamas con la Organización Estado Islámico (Daesh) (The Jerusalem Post, 20I4). La criminalización de la protesta y el descrédito de las prácticas autónomas han sido otras batallas más que los pueblos en resistencia han tenido que librar a lo largo de su historia. 
Un par de ejemplos de lo anterior pueden ser la intifada palestina, en 1987, por un lado, y la batalla de Ocosingo, en 1994, dos casos que, aunque diferentes por las circunstancias donde se expresa una forma particular de violencia libertaria, tienen resonancias en tanto que en ellos diversos grupos autónomos enfrentaron a dos Ejércitos regulares (en el caso palestino, el Ejército israelí en calidad de ocupante; en el caso zapatista, el Ejército mexicano en calidad de represor), existiendo en ambos contextos desigualdad, marginación, provocación armada, intimidación y otras formas de violencia psicológica perpetradas por las fuerzas de seguridad del Estado. ${ }^{12}$

En segundo lugar, se encuentra el desentendimiento de los acuerdos jurídicos entre el Estado y los pueblos marginados. Para muchos pueblos en resistencia, las armas no serían una opción si la ley funcionara, pero los acuerdos, tratados y otros instrumentos han sido mecanismos que más que ayudar a la emancipación la han enterrado o, al menos, han coadyuvado directa e indirectamente a igno-

12. Aunque hay contundentes diferencias entre la intifada palestina y la batalla de Ocosingo, cada uno de estos fenómenos es un ejemplo de violencia libertaria. En diciembre de 1987, una camioneta que transportaba obreros palestinos sufrió la embestida de un camión militar israelí y cuatro trabajadores perdieron la vida. En la ciudad de Gaza y los alrededores se corrió rápidamente la voz y miles de personas salieron a las calles a manifestarse contra los ocupantes, enfrentándose a pedradas contra las tropas israelíes a través de la organización de varios comités que formaron una conducción centralizada y fueron coordinando y dirigiendo la movilización desde los barrios y los campamentos. En lo concerniente a la batalla de Ocosingo, la madrugada del I de enero de 1994, tras un enfrentamiento de horas entre zapatistas y el destacamento policial de Ocosingo, unos seiscientos zapatistas ocuparon el ayuntamiento de la ciudad, algunas de cuyas dependencias fueron incendiadas, al igual que la sede de los juzgados. Desde la estación local de radio, los zapatistas lanzaron sus peticiones revolucionarias de reforma agraria, mejoras sociales y dimisión del Gobierno presidido por Carlos Salinas de Gortari. A las 15:50 horas del día 2 de enero, entraron a la población tres batallones de infantería del Ejército mexicano que participarían en una operación envolvente que obligaría a muchos zapatistas a refugiarse en el mercado de la localidad. A diferencia de lo sucedido en la intifada palestina, la gente de la ciudad estaba encerrada en sus casas, atemorizada, no sin antes haber estado a favor de la causa zapatista, según narran diversas fuentes impresas. 
rarla. Para profundizar, se puede citar a Linda Quiquivix (2013), quien explica cómo la cooperación en el ámbito del derecho entre Estados se ha convertido en un elemento más de las lógicas de dominación usadas por estos a nivel transnacional. La autora pregunta sobre la utilidad de las reclamaciones legales, nacionales e internacionales, para las luchas emancipadoras, cuando el concepto de ley que se usa en el denominado "Occidente" limita y determina lo que los sujetos en lucha son capaces de hacer o no.

Aquí, se debe problematizar sobre la existencia de una autoridad soberana a la que se le confiere la salvaguarda de "nuestros derechos", y la cual se encarga de la concesión, el reconocimiento y el control de los mismos, en contraste con los ejercicios de soberanía y los ejercicios de autodeterminación. Respecto a esto, Quiquivix sostiene que no hay un vínculo inherente entre la aplicación de la ley y la consecución de la justicia en Occidente si la ley con la que se pretende alcanzar dicha emancipación es la misma con la que el Estado reproduce las relaciones de dominación, privatiza la tierra y crea "ambientes propicios para algunos por encima de pueblos enteros" (Quiquivix, 2013, p. 2).

Entonces, por más que se presente a la ley como una herramienta flexible para defenderse y "reclamar derechos", esta termina siendo una táctica contra la autodeterminación, la cual, por definición, no puede ser otorgada por un organismo externo. Para efecto de esto, se pueden citar los casos de los Acuerdos de Oslo, en 1993, ${ }^{13}$ y los Acuerdos de San Andrés Larraínzar, en 1997, ${ }^{14}$ dos ejemplos de cómo la ley del Estado ha servido como táctica de sometimiento y

13. El texto completo de los acuerdos está disponible en: http://cis.uchicago. edu/sites/cis.uchicago.edu/files/resources/CIS-0902 I 3-israelpalestine_381993DeclarationofPrinciples_OsloAccords.pdf

14. El texto completo de los acuerdos está disponible en: http://biblio.juridicas. unam.mx/libros/I/I/I2.pdf 
no como instrumento de defensa de los derechos de quienes la suscriben.

En el caso de los Acuerdos de Oslo, es útil saber que su firma representó la aprobación de un modelo que relegó la soberanía palestina, en el cual la fórmula "territorios por paz" no hizo otra cosa que presuponer que Israel tenía "territorios" que estaría dispuesto a conceder a los "árabes", y que estos -considerados como responsables directos del estado de guerra- podrían garantizar a Israel la paz que había anhelado durante mucho tiempo. En el caso de los Acuerdos de San Andrés, en una dinámica diferente, este instrumento representó un escenario para romper la concepción monoétnica del Estado mexicano en que se buscaba un pacto social renovado donde se otorgaran derechos no sólo a los individuos y a los ciudadanos, sino también a las colectividades. Aunque esto suponía un cambio en las formas de relacionarse entre el Estado y los pueblos indígenas, el Gobierno mexicano no cumplió con los acuerdos, y los pueblos indígenas, a través del Congreso Nacional Indígena, acordaron construir autonomías "desde abajo" que tendrían gran repercusión global, tales como las que se mencionarán a continuación.

\section{Tres ejemplos de glocalidad zapatista}

El primer ejemplo de autonomías desde abajo en el zapatismo es el de las Juntas de Buen Gobierno y los Caracoles, unos de los niveles de gobierno en los que está dividido el sistema autónomo zapatista. De acuerdo con uno de sus comunicados (EZLN, 2003), los zapatistas crearon estas formas de organización para llevar a la práctica su autonomía de gobierno y evitar la imposición de proyectos externos por parte de la sociedad civil nacional e internacional.

Las juntas, en este sentido, son las instancias donde se hace la gestión política institucionalizada de las comuni- 
dades indígenas, se toman decisiones consensuadas, se destinan recursos, se promueven proyectos y se establecen relaciones con otras instancias zapatistas. Así, no sólo existe una reapropiación del espacio-tiempo de las relaciones sociales de la comunidad, sino también se reproduce un nuevo contrato social de carácter intersubjetivo que se materializa en cada uno de los procesos y decisiones que se toman entre el EZLN, las comunidades indígenas civiles zapatistas y la sociedad civil, en los cuales las comunidades indígenas son el actor principal en tanto las juntas sólo pueden integrarse con miembros de las propias comunidades.

En este esquema, y en el tenor de la lógica de la violencia libertaria, el EZLN participa como defensor armado de los territorios para oponerse a los ataques del Ejército federal mexicano y los grupos paramilitares antizapatistas e, incluso, a los cárteles de droga aledaños, asumiendo que pese a la existencia de una declaración de guerra contra el Estado mexicano, los zapatistas no buscarían la desaparición del mismo.

A su vez, la sociedad civil nacional e internacional que simpatiza con el zapatismo participa apoyando al desarrollo de proyectos locales a través de los Caracoles, espacios político-culturales de encuentro entre las comunidades indígenas zapatistas y la sociedad civil, lo cual proyecta internacionalmente esta serie de prácticas locales, materializando la noción de glocalidad. A través de esos enlaces, entonces, es que las comunidades nombran a sus autoridades locales para que cumplan sus mandatos, a sabiendas de que si no lo hacen serán revocados.

A través de lo anterior, se fortalecieron los vínculos de solidaridad entre otras comunidades locales de distintas etnias y se articularon unidades mayores que comprendían otros municipios. De hecho, los Caracoles y las Juntas de Buen Gobierno son para algunos intelectuales (Casanova, 2003) algunos de los ejemplos de autonomía contra la globa- 
lización más avanzados del tiempo actual porque articulan y proponen un proyecto alternativo de organización que, arrancando de lo local y lo particular, pasa por lo nacional y llega a lo universal; dejan a sus integrantes toda la responsabilidad de cómo trazar el recorrido y la autonomía; se trata de programas de acción, de conocimiento, de perseverancia y dignidad para construir un mundo alternativo; y, algo muy importante, se da pie en ellos al autogobierno, que pugna por no tomar el poder del Estado, sino ejercer el que tienen y siempre han tenido (Martínez, 2006, p. 24).

Un segundo ejemplo de glocalidad en el zapatismo es el de la banca. Aunque pudiera ser contradictorio hablar de un "sistema financiero" al interior de un movimiento antisistémico como es el "mandar obedeciendo", la banca zapatista representa una de las actividades anticapitalistas y autónomas más importantes en lo que se refiere a la distribución y uso de los recursos del movimiento. Se compone de dos bancos anticapitalistas, el BANPAZ (Banco Popular Autónomo Zapatista) y el BANAMAZ (Banco Autónomo de Mujeres Zapatistas), los cuales sirven para administrar las donaciones de la sociedad civil y para dar muestra de la disponibilidad de recursos para proyectos que designen las juntas. El Centro de Documentación sobre Zapatismo (CEDOZ, 2009) informa que, al igual que las otras bases del gobierno autónomo, el objetivo de la banca zapatista es lograr la autosuficiencia en condiciones de extrema precariedad, donde uno de los principales problemas es el de la salud. Así, la necesidad urgente de dinero para enfrentar un problema de salud grave, por un lado, y el incremento del fenómeno del "coyotaje", ${ }^{15}$ por el otro, conformaron un escenario donde se discutió la idea de formar un pequeño banco para cubrir las necesidades de urgencia de salud, es

I5. Proporcionar medicinas o materiales de curación a personas de manera ilegal. 
decir, de aquellos casos graves que no pudieran ser cubiertos por los servicios de salud autónomos.

En este tenor, las comunidades zapatistas decidieron crear estos bancos para tener disponibilidad de préstamos con bajo interés para emergencias. Con dichos intereses se incrementaría el fondo del banco para hacer otros préstamos y beneficiar a más gente. El interés acordado para préstamos por motivos de salud ha sido del $2 \%$ mensual, y para proyectos colectivos, sociedades o cooperativas del $5 \%$ (CEDOZ, 2009).

En un principio, se pensó también en préstamos para proyectos individuales, pero, explican, "nos dimos cuenta que eran para puro negocio y los suspendimos, quedando sólo vigentes los préstamos por problemas de salud y para después los préstamos para proyectos colectivos" (CEDOZ, 2009). Lo importante aquí es que ninguna decisión es individual, y que los pueblos son los que analizan cada paso y van decidiendo lo que les conviene, en un claro ejemplo de reapropiación de los símbolos de opresión del capitalismo, como lo pueden ser el dinero, el interés y la misma banca.

Un tercer ejemplo ha sido "la Escuelita", una convocatoria hecha por los zapatistas para crear un espacio de compartición de "la palabra del movimiento", sus demandas y principios. ${ }^{16}$ La Escuelita se planeó como un espacio para que invitados de todo el mundo (entre quienes asistieron algunos alumnos palestinos) (Rohana, 2014) pudieran experimentar cómo viven los zapatistas, cómo organizan y gobiernan sus comunidades y entendieran por qué y cómo resisten

16. Los principios son obedecer y no mandar. Representar y no suplantar. Bajar y no subir. Servir y no servirse. Convencer y no vencer. Construir y no destruir. Proponer y no imponer. Las inspiraciones filosóficas son: "la construcción de un mundo donde quepan muchos mundos" y "para todos todo, nada para nosotros". Sus demandas son trece (diez del zapatismo y tres nacidas de su fusión con la sociedad civil): techo, tierra, trabajo, salud, alimentación, educación, independencia, democracia, libertad, justicia, cultura, información y paz. El principio fundamental del gobierno autónomo es "mandar obedeciendo". 
al Gobierno mexicano. En la Escuelita se replantean los conceptos de educación, maestro, libertad, resistencia, el papel de la mujer, entre otros temas importantes que se enseñan a través de la experiencia de la vida ordinaria de las comunidades y sus narrativas.

Los zapatistas informaron que todo lo que se necesitaba para ser un alumno de la Escuelita era apertura epistémica, es decir, "mirar y aprender". El lugar de la enseñanzaaprendizaje era la comunidad donde viviría, comería, estudiaría y haría lo que todos y todas hacen. El alumno tendría un votan (tutor), quien explicaría la historia, éxitos y errores del movimiento, y quien sería el responsable del invitado en todo momento. En resumen, y en palabras de Jorge Alonso (2014), "lo que se espera encontrar en la escuelita es la vida cotidiana, sus problemas, la manera de resolverlos y la manera en que se están construyendo otros mundos fuera de la capital y el Estado" (p. 7).

El proyecto de la Escuelita, como parte de un proceso autónomo, vela por una educación que se distancia de los planes oficiales del Estado mexicano, y establece una glocalidad interesante en la cual la Escuelita no va al extranjero (aunque se le conoce y se le promueve), sino que es el estudiante, nacional o extranjero, aquel que tiene que asistir a los cursos, estudiar y aprobar los exámenes que aplican los zapatistas y sus guardianes (votán).

En este proyecto, los zapatistas han conservado algunas materias, como las matemáticas, y han agregado otras, como el medioambiente, esto a través de sus propios libros de texto y la capacitación de sus propios profesores. En lo que se refiere a la Escuelita, esta incluye la enseñanza y compartición del día a día en el trabajo, el ganado, el cafetal comunitario, la leña, entre otras actividades relacionadas con el gobierno, la autonomía, la resistencia y el papel de las mujeres en el sistema, quienes narran que la revolución ciertamente había comenzado en casa porque ellas no 
participaban en la organización de la comunidad pues se pensaba "que sólo servían para el hogar o cuidar los hijos" (Eloísa, 2013, pp. 6-7), esto hasta que comenzaron a participar en las Juntas de Buen Gobierno, la comandancia y otras actividades donde todos y todas podían aportar, tal como lo ha mostrado el ejemplo de la comandante Ramona, todo un pilar y un ejemplo de lo que algunos investigadores llaman "el feminismo zapatista" (Marcos, 2014).

\section{Tres ejemplos de glocalidad palestina}

Sharaka (“asociación” en árabe) es un proyecto autónomo palestino fundado por Aisha Mansour, el cual emula una granja urbana que distribuye alimentos a la gente a través de una relación cercana con el recolector. No muchas personas pueden hacer granjas en la ciudad, mucho menos cuando la ciudad está ocupada militarmente. Por tal motivo, un grupo de jóvenes se ha dado a la tarea de acercar los alimentos cultivados en sus granjas directamente a la mesa de los palestinos que viven en Ramallah, la capital palestina, esto mediante su eslogan por excelencia: "comida local para la gente local".

El trabajo de Sharaka se hace mediante una gestión completamente voluntaria que pretende conectar el día a día de los palestinos agricultores con el día a día de los palestinos que viven en contextos urbanos. Los agricultores y recolectores usan sus propios recursos locales y buscan apoyarse mutuamente a través de una economía local en la que prácticamente se convierten no sólo en productores sino también en distribuidores de sus propios alimentos, explotando la poca tierra disponible para el cultivo. El trabajo de Sharaka también se basa en los lazos de unidad, solidaridad y comunidad palestina que intentan convencer a los palestinos de Ramallah para que asistan a sus mercados y compren sus productos (o se haga trueque si no se tiene 
dinero) con el fin de enfrentar la "inundación" de productos baratos provenientes de Israel (Sharaka, 2014).

El grupo que lidera Mansour se ha negado a aceptar cualquier tipo de ayuda internacional para apoyar su trabajo debido a que ello es un sistema que no funciona pues muchas veces las organizaciones internacionales buscan lucrar con la imagen de la causa y su victimización. En palabras de Mansour:

[...] la gente no quiere lástima, quiere desarrollo, y la gente local que conoce a su comunidad, que quieren establecer y respaldarla, que hacen las cosas bien, saben que el mutuo apoyo es una buena forma de desarrollarse y no de desarrollarse como el exterior quiera (Mansour, 2012, p. 96).

Lo anterior es importante porque Sharaka es uno de los pocos proyectos que están desarrollando propuestas de consumo local sin necesidad de patrocinadores externos. Basta recordar que los antes mencionados Acuerdos de Oslo transformaron a la Autoridad palestina -el Gobierno palestino creado como resultado de dichos acuerdos- en un actor totalmente dependiente para tener un presupuesto de los donantes internacionales. Hoy en día, la Autoridad nacional palestina tiene cerca de 3000 millones de dólares de deuda, lo cual deja claro la poca flexibilidad que hay en la economía cuando se produce y se consume en un sistema que no permite generar recursos propios para pagar sueldos, mantener sectores públicos dignos o servicios de salud en buen estado.

En este sentido, en palabras de Nora Lester Murad, una voluntaria en Sharaka y cofundadora de otra organización autónoma llamada Dalia:

[...] no se debe jugar con el sector de la alimentación, pues una organización palestina que aboga por un mejor uso de los recursos locales, la disminución de la dependencia del exterior y el desarrollo local ciertamente satisface los objetivos palestinos (Lester, 2014). 
Ella ha dicho también que si bien la ayuda internacional ha llevado algunos beneficios a la sociedad palestina (incluyendo los empleos y la creación de instituciones de base), ha sido en gran parte destructiva porque no ha ayudado en la reivindicación de derechos e incluso ha ayudado a hacer más difícil la resolución del conflicto, en tanto que el dinero corrompe a las personas.

Lo más importante en esta clase de trabajo ha sido que en esta nueva conceptualización de la alimentación, de la granja o la villa, la comunidad decide por la misma comunidad, y que estas decisiones provocan que las relaciones sociales tradicionales al interior de la aldea no sólo se mantienen sino que se convierten en el elemento potencial que permite la resistencia a la ocupación israelí, teniendo en cuenta las transformaciones espaciales y urbanas que esta ocupación ha tenido en los últimos años.

Así, una práctica local como la de Sharaka invita a que los palestinos vuelvan al sistema de desarrollo sostenible y autónomo que caracterizó a la primera intifada, a finales de 1980, en la cual el desarrollo, el trabajo, la lectura y cualquier proyecto que la comunidad tenía necesariamente implicaban un enfoque que reforzara la existencia de Palestina y la resistencia contra la ocupación israelí, superando el dilema de la confiscación ilegal de tierras en un contexto internacional marcado por el internamiento cada vez mayor de empresas transnacionales, cuyos recursos, en mucho casos, implican capital financiero israelí (Kestler, 2013).

Un segundo ejemplo de glocalidad en Palestina es el llamamiento de la sociedad palestina a participar en el movimiento Boicot, Desinversión y Sanciones contra Israel, mejor conocido como BDS. Este movimiento es un instrumento cívico y pacífico que se ha convertido en una forma eficaz de movilización social y presión política contra la permisividad de los Gobiernos con Israel. El BDS nace de los mismos palestinos: ellos son los que coordinan, los que 
dicen qué se puede hacer desde el interior y desde cualquier trinchera fuera de Palestina.

El fracaso de los Acuerdos de Oslo, la omisión de las declaraciones de la ONU, las intervenciones militares en Gaza y la falta de compromiso de los actores internacionales han producido este llamamiento del pueblo palestino hacia los pueblos del mundo. Se trata de una campaña contra el racismo y la segregación que se mueve flexiblemente con el margen que ofrecen los derechos humanos y el derecho humanitario internacional, donde la sociedad civil palestina demanda a las organizaciones de la sociedad civil internacional y a las personas conscientes de todo el mundo imponer amplios boicots e implementar desinversiones contra Israel, de manera similar a aquellas aplicadas a Sudáfrica en la era del apartheid (Barakat, et al., 2005). A través de esta campaña, los palestinos demandan que los ciudadanos conscientes presionen a sus respectivos Estados para que impongan embargos y sanciones contra Israel, pero, más allá de esto, se invita a los propios ciudadanos a apoyar esta demanda por medio de un consumo responsable.

El BDS está concebido como una acción glocal y antisistémica pues prevé que cada práctica local puede coadyuvar a minimizar las ganancias y la riqueza con las que Israel financia su ocupación militar en Palestina. Casualmente, si se voltea a ver la lista de empresas y productos que se pide no consumir porque colaboran con la ocupación israelí, se podrá notar que se trata de las empresas que controlan un gran porcentaje de los intercambios a nivel mundial, y que suelen ser las que se expanden más rápidamente en los espacios públicos de muchos países en el mundo. ${ }^{17}$

Una crítica que ha recibido el BDS de sus detractores es que se trata de una medida contra judíos y contra los ciu-

17. La lista está disponible en: http://www.bdsmovement.net/activecamps/ consumer-boycott 
dadanos de Israel; no obstante, se puede decir que no es así porque entre aquellas comunidades hay quienes luchan por las mismas metas de los palestinos que impulsan el BDS, siendo el objetivo de la campaña tejer redes de indignación y esperanza entre la comunidad internacional contra el poder sionista y colocar el BDS como una herramienta de acción política que incentive nuevas formas de acción, como el boicot académico, que invita a los intelectuales de reconocidas universidades en el mundo a no tratar con universidades israelíes (Palestinian Campaign for the Academic and Cultural Boycott of Israel, 2004).

Un tercer ejemplo de glocalidad se encuentra en la Comunidad Palestina de Museos, la cual se trata de una red de cincuenta y un museos que intenta preservar y proyectar la cultura, la historia y el presente de los territorios palestinos ocupados. En Palestina no todos los museos funcionan, pero un grupo de interesados, algunos asalariados por la Autoridad nacional palestina, y otros voluntarios, se encargan de darles vida, proyección y mantenimiento a cada uno de ellos. Uno en particular, el Museo Palestino, está siendo terminado en Berzeit, a 7 kilómetros de Ramallah, y su apertura oficial será a principios de 2016. Utilizando un enfoque innovador en el diseño del espacio, el proyecto rompe con tradiciones en la práctica museística, pues expone el arte y la cultura palestina en un tono de conversación con el mundo y las ideas para el futuro.

La apuesta visual del museo está construyendo una red de asociaciones y satélites en el extranjero con fines de extender más allá de las fronteras geográficas de Palestina la historia y la cultura de este pueblo, además tomando en cuenta que más de la mitad de los palestinos en el mundo se encuentran fuera de Palestina. Al principio, se pensó que el nombre del museo podría ser "Museo de la Memoria de Palestina", pero dicha idea fue abandonada cuando la segunda intifada estalló en septiembre de 2000 y la gente 
comenzó a acercarse a la idea del museo desde una perspectiva más amplia, de fronteras abiertas, con lo que no necesariamente debía iniciar o detenerse en la nakba, sino mirar a los palestinos antes y después de eso (Meador, 2014).

La red de museos de Palestina cuenta con grandes piezas como el Museo de Mahmoud Darwish, también en Ramallah, donde se pueden apreciar las obras del poeta nacional palestino, o el Markaz athurat alfilastini fi bait Lehem (Centro para la Difusión de la Herencia Palestina), el cual muestra una gran gama de textos textiles y de vestidos para mujeres que son garantes de la identidad indígena palestina. ${ }^{18}$

Regularmente, las exposiciones, talleres, muestras de cine, conferencias y otras actividades artísticas involucran a más palestinos para que se interesen en las actividades de la comunidad. El término "comunidad de museos" es algo que la gente está tratando de desarrollar porque este campo está tan fragmentado (por el muro israelí, los checkpoints, y otros factores) que a veces parece que cada museo es totalmente independiente del resto. El problema cotidiano es que no hay comunicación, se depende de la electricidad que Israel controla. Así que lo que se trata de hacer es encontrar un terreno común donde todos puedan conocer y compartir, encontrar sinergias, espejos, y sentimientos como pueblo, o como dice Jack Persekian, director del Museo Palestino, "encontrar un lugar seguro para las ideas peligrosas donde no se abandone la historia en una vitrina sino donde se abra un punto de referencia para seguir luchando" ( $A l$ Mathaf al Filastini, 2014).

En una geografía como la palestina, donde hay una gran fragmentación y la gente no puede tener contacto entre sí, se tiene que pensar en cómo se pueden crear vínculos, es decir,

18. El sitio en internet es: http://www.palestinianheritagecenter.com/, y fue consultado el 2 de diciembre de 2014 . 
cómo se puede hablar de conceptos e ideas y cómo se puede colaborar con los palestinos y con los que siendo solidarios con Palestina no comparten la misma área geográfica. Por eso se tiene que pensar en apertura y en convertir cada centro de una pequeña ciudad, villa, barrio, restaurante, mercado o casa en una extensión de cualquier museo de la tierra palestina.

Reflexiones finales: la vida como resistencia, la autonomía como medio y la palabra como compañera

En la entrada del consulado palestino en México hay una fotografía que retrata los rostros de Emiliano Zapata y Abd al-Qadir al-Husayni, dos héroes de las revoluciones mexicana y palestina, sucedidas en 1915 y 1948, respectivamente. Dicha fotografía, que implica la fraternidad en ambas luchas por la justicia y la dignidad, ha estado presente incluso desde años atrás, cuando los sombreros, carretes, rifles y bigotes dominaban la estética de la Revolución mexicana.

Actualmente, y después del recorrido hecho en el presente texto, se puede decir que las acciones glocales, compuestas principalmente por la autonomía y las prácticas locales autogestivas, se han proyectado como estrategias de resistencia a la globalización neoliberal debido a que se han tenido que enfrentar diversas formas de opresión y homogenización económica por parte de los dueños del capital y sus ejércitos.

Las resonancias que encontramos entre las luchas, es decir, en las formas locales de producción, distribución y consumo de recursos, las formas diferentes de producción y reproducción del espacio, la reapropiación de la palabra y el discurso, el uso del acto como utopía, la desobediencia epistémica a los marcos legales establecidos como herramientas de dominación, el uso de violencia libertaria, e 
incluso las contradicciones en su relación con el Estado, sus modelos financieros y las empresas transnacionales que promueve, son factores que permiten seguir hablando de la fraternidad, de la traducción cultural y de "lo común" y lo resonante que hay entre ellas, en tanto siguen en la construcción de su propia dignidad y justicia social. Tanto en Chiapas como en Palestina, la revolución comenzó en el hogar cuando muchas mujeres comenzaron a desempeñar acciones vitales para la resistencia, la autonomía y la narrativa de sus propias luchas, tal como lo demuestra el protagonismo que en cada ejemplo enunciado anteriormente han tenido las mujeres en sus respectivas prácticas locales.

Un punto importante a notar es que tanto zapatistas como palestinos se encuentran entre la industria de la militarización (con la cooperación militar entre México e Israel) y de la pacificación (con el intento de un puñado de ONG que buscan moldear la paz en el terreno, pero que victimizan e idealizan las causas), situación que se agrava si pensamos que el contexto de esto es el neoliberalismo y la guerra contra la humanidad.

Lo anterior va encaminado a hacer notar que zapatistas y palestinos comparten una nakba, una catástrofe, la cual sortean en gran medida con la ayuda y el involucramiento de la misma población, su ética, educación, compromiso, solidaridad y necesidad de mutuo apoyo. En este sentido, se podría decir que las Juntas de Buen Gobierno y los Caracoles, del lado zapatista, fueron el resultado de la reorganización interna del colectivo frente al contexto adverso al que se enfrentaba, tal como lo ha sido el BDS en el caso palestino, dos ejemplos de claro alcance global por la dimensión de sus objetivos y por la clara desobediencia epistémica que implica su andamiaje cotidiano ante el fracaso de iniciativas jurídicas donde el Estado, o bien no cumplió con los acuerdos, como en el caso de San Andrés, 
o bien los usó para fortalecer la ocupación, como en el caso de Oslo, ambos en la década de los noventa.

El fracaso de las herramientas jurídicas o su mal funcionamiento han dado pie a una consideración final, es decir, a la del rescate de la vida ordinaria como acto político contestatario. La misma vida, como contestación a una política de muerte o necropolítica, siguiendo a Mbembe (2003), es una posibilidad en sí misma de crear epistemes y de (re)producir las formas más complejas de resistencia en cualquier tipo de ambientes.

La vida como política, como resistencia y utopía, ofrece una mirada sobre las maneras en las que la gente sin partidos políticos, la gente común, ha enfrentado a una plétora de grupos encargados de recrear el espectáculo de la violencia y la muerte para dividir, atemorizar y disuadir a la población. En este sentido, autores como Bayat (2013, p. 42) reconocen, en una posición muy cercana al zapatismo, que la gente, mientras vive, también resiste y crea mecanismos diferentes para contar la historia y hacer política en las calles y el barrio, en los cuales la comunidad es el eje fundamental del bien personal.

Los proyectos descritos en este texto han tenido como base a todas esas personas de la vida ordinaria que al ser marginadas por la globalización neoliberal han tratado de apropiarse de algunas ventajas tecnológicas de esta, de sus leyes, de sus posibilidades de conectarse con el mundo, y han impulsado acciones de cambio con un impacto global, sea por las formas en las que se habla de ellas, se emulan o simplemente se conocen, ayudando a que se materialice una red de proyectos resonantes que forma un proceso contrario a la homogenización neoliberal.

En las formas anteriores no hay colectivos, manifiestos o líderes claros, sino una serie de acciones prolongadas por parte de individuos, familias y grupos dispersos que se juntan para buscar la adquisición o demanda de necesida-

\section{6}


des básicas (tierra, comida, refugio, democracia, consumo, historia, trabajo, educación, oportunidades de negocio o espacio), pues en la actual coyuntura han sido las personas, y sólo las personas viviendo en grupo y para el grupo, las que han hecho posible un "caracol", un banco en la selva o un museo en el desierto, y ellas se convierten en actores construyendo el escenario futuro a partir del presente, sin olvidar su cultura y su palabra.

De lo anterior, otro punto importante es aquel donde las labores ordinarias de estas autonomías se vuelven extraordinarias, es decir, cuando logran conectarse con eventos de protesta masivos donde interactúan con actores políticos organizados, jerarquizados y experimentados, críticos de las redes de poder y que se preocupan por otros niveles de lucha (sindical, indígena, feminista, estudiantil), y entonces se logra reconfigurar una nueva expresión política desbordante o tal vez conocer la emergencia de nuevos actores, pero diferentes en las formas de hacer y entender la política. Esto ha pasado cuando el zapatismo y los palestinos, cuando Gaza y Chiapas, con sus actos y sus palabras, alcanzan resonancias con intelectuales como los que se han citado en este trabajo, así como con movimientos sociales como los de Ferguson o Ayotzinapa, y cuando parece que esas causas locales, por momentos, se vuelven globales.

La participación de la gente ordinaria en concentraciones masivas de larga envergadura al lado de afiliados o simpatizantes de movimientos sociales, ONG, sindicatos, colectivos, entre otros, con los cuales no existen precisamente coincidencias ideológicas sino sólo resonancias y, en muchos casos, disensos, es un eslabón que puede llegar a ser muy importante para proyectos de largo aliento en la misma protesta desde abajo, en tanto los regímenes represores se preocupan mayoritariamente por cooptar a los actores que históricamente han sido más visibles y que se han conside- 
rado como los líderes de las formas de contestación masivas y otros ejercicios de desobediencia social.

Por tal motivo, elementos como la cotidianeidad, la fluidez, la creatividad y la espontaneidad de la gente de a pie son blancos difíciles para los Gobiernos represores que invierten su tiempo y recursos de inteligencia en localizar cabecillas, líderes o personajes clave en el andamiaje de la movilización social.

Con el paso del tiempo, las glocalidades, sostenidas por la práctica autónoma y autogestiva, pueden aprovechar oportunidades políticas para tender puentes y adoptar múltiples formas de organizarse de manera muy diferente a como lo haría, por ejemplo, un sindicato o una ONG, siendo las asambleas un pilar que sostuvo (y que sigue sosteniendo) tanto las protestas ciudadanas como los proyectos locales de autodeterminación económica y social, incluso en los días más tensos y de mucha incertidumbre de los procesos revolucionarios. En este sentido, el zapatismo y la resistencia palestina han dado buenos ejemplos y muestras de ello en los últimos veinte años.

Fecha de recepción: 06 de enero de 2015

Fecha de aceptación: 19 de junio de 2015

Bibliografía

\section{8}


, y Alonso, C. (20I5). En busca de la libertad de los

Bibliografía de abajo: la demoeleuthería. Guadalajara: Universidad de Guadalajara.

Barat, F., y Gómez, L. (20 I4). BDS por Palestina: el boicot a la ocupación y el apartheid israelíes. Madrid: Ediciones del Oriente y del Mediterráneo.

Bayat, A. (2013). Life as politics: how ordinary people change the Middle East. Ámsterdam: Amsterdam University Press.

Castellanos, L., y Jiménez, A. (2007). México armado. 19431981. Mexico, D. F.: Ediciones Era.

CEDOZ (2009). "Insólito banco anticapitalista en la selva Lacandona”. Centro de Documentación sobre el Zapatismo. México: CEDOZ. Recuperado de: http://www.cedoz.org/ site/content.php?doc $=892 \&$ cat $=8 \mathrm{I}$

Deleuze, G., y Guattari, F. (1972). Capitalisme et Schizophrénie I. L’Anti-Édipe. París: Minuit.

Eloisa (20I3). “Introducción”, en, Participación de las mujeres en el gobierno autónomo. Cuaderno de texto de primer grado del curso “La libertad según I@s zapatistas” (p. 6). Chiapas, México. Recuperado de: https://chiapasbg.files. wordpress.com/2013/09/mujeres-es.pdf

Eqeiq, A., et al. (20I3). Writing the indigenous: Contemporary mayan literature in Chiapas, Mexico and Palestinian literature in Israel (tesis doctoral inédita). University of Washington: Washington.

EZLN (2003). Chiapas: La treceava estela. México: EZLN. Recuperado de: http://palabra.ezln.org.mx/comunicados/2003/2003_07_a.htm

(dir.) (2009). Pronunciamiento de los zapatistas frente a la masacre del pueblo palestino. Material audiovisual. Recuperado de: https://www.youtube.com/ watch? $v=w R 9 D n Q H V P I Y$

Forcades, T., y Vivas, E. (20I3). Conversación entre Teresa Forcades y Esther Vivas: sin miedo. Barcelona: Icaria. 
Bibliografía
Foucault, M. (1977). Discipline and punish: The birth of the prison. Nueva York: Pantheon Books.

Fukuyama, F. (1992). The end of history and the last man. Nueva York: Free Press, Maxwell Macmillan Canada, Maxwell Macmillan International.

Giraldo, O. F. (20I3). Utopías en la era de la supervivencia: una interpretación del buen vivir. Chapingo: Universidad Autónoma de Chapingo.

González, P. (2003). "Los caracoles zapatistas: redes de resistencia y autonomía”. Revista Memoria, (176), I4-19.

Habibi, S. (27 de enero de 2009). "Palestinian economy: Foundation of a state or common burden?". The Electronic Intifada. Recuperado de: https://electronicintifada. net/content/palestinian-economy-foundation-state-orcommon-burden/8026

Holloway, J. (2005). "Zapatismo urbano”. Humboldt Journal of Social Relations, 29(I), I68-I78.

Johnson, J., y Quiquivix, L. (8 de junio de 20I3). "IsraelMéxico: cooperación para aplastar a los zapatistas”. El Ministerio. Recuperado de: http://www.elministerio. org.mx/blog/2013/06/mexico-israel-ezln-cooperacionmilitar/

Kestler, J. (28 de febrero de 2013). "Farmers' markets bypass foreign aid to Palestine". The Electronic Intifada. Recuperado de: https://electronicintifada.net/content/ farmers-markets-bypass-foreign-aid-palestine// 2240

Lester, N. (20I4). "Dalia Association”. Dalia Association. Recuperado de: http://www.dalia.ps/

Malacrida, L. E. (1998). La inseguridad social de los argentinos: utopía y resignación. Buenos Aires: Editorial de Belgrano.

Mansour, A. (20I2). Impact of Post Oslo Aid Interventions on the Palestinian Agricultural Sector (tesis enviada en cumplimiento parcial del Master in International Cooperation and Development). Bethlehem University: Palestina. Recuperado de: http://www.apis.ps/up/ I 344237 I 20.doc 
Marcos, S. (8 de septiembre de 20 I4). "El feminismo zapatista, Bibliografía otra trinchera para las mujeres”. Observatorio Género y Equidad. México: Observatorio Género y Equidad. Recuperado de: http://www.observatoriogeneroyliderazgo. cl/index.php/bloc-mainmenu-9/26-novedades/768Ifeminismo-zapatista-otra-trinchera-para-las-mujeres Márquez-Fernández, Á. (2006). Globalización neoliberal y filosofia intercultural. México: Universidad de Navarra. Recuperado de: http://www.unav.es/gep/SeminarioMarquez.html

Mbembe, A. (2003). "Necropolitics”. Public Culture, I5(I), II -40 .

Melucci, A. (1996). Challenging codes: collective action in the information age. Nueva York: Cambridge University Press.

(1999). Acción colectiva, vida cotidiana y democracia. México, D.F.: El Colegio de México.

Milenio (26 de noviembre de 20I3). "México e Israel firmarán acuerdos durante la visita de Peres". Milenio Noticias. Recuperado de: http://www.milenio.com/politica/ Mexico-Israel-firmaran-acuerdos-Peres_0_197380597. html

Olson, M. (197I). The logic of collective action; public goods and the theory of groups. Cambridge: Harvard University Press.

Pacheco, L., et al. (20I I). "Los pueblos indios en los libros de texto gratuitos". Revista Mexicana de Investigación Educativa, I6(49), 525-544.

Palestinian Campaign for the Academic and Cultural Boycott of Israel (2004). Call for academic and cultural boycott of Israel. Ramallah, Palestina: PACBI. Recuperado de: http:// pacbi.org/etemplate.php?id=869

Pappé, I. (2006). The ethnic cleansing of Palestine. Oxford: Oneworld. 
Bibliografía
Peled-Elhanan, N. (2012). Palestine in israeli school books: ideology and propaganda in education. Londres: I.B. Tauris.

Quiquivix, L. (20I4). "Law as tactic: Palestine, the zapatistas, and the global exercises of power". Al Majdal Badil Research Center Magazine, (55), 34-40. Recuperado de: http://www.badil.org/phocadownload/Badil_docs/ publications/al-majdal-55.pdf

Raz, J. (1986). The morality of freedom. Oxford: Clarendon Press, Oxford University Press.

Ricco, M. (I 0 de febrero de 1995). "El presidente mexicano califica de terroristas a los dirigentes zapatistas y ordena su captura”. El País. Recuperado de: http://elpais.com/ diario//995/02//0/internacional/7923708/8_8502I5. html

Rivera, J. (ed.) (20I4). Crimen organizado y autodefensas en México: el caso de Michoacán. México: Programa de Cooperación en Seguridad Regional Friedrich-EbertStiftung (FES).

Rohana, S. (I0 de agosto de 20I4). "Solidarity with Gaza from the Mexican periphery". Al Jazeera English. Recuperado de: http://www.aljazeera.com/indepth/ opinion/20 I 4/08/solidarity-with-gaza-from-mexican-20I4899492690432.html

Santos, B. (20I I). "Epistemologías del sur". Utopía y Praxis Latinoamericana, I6(54), I7-39.

— (16 de noviembre de 2014). "Carta a las y los jóvenes de México”. La Jornada. Recuperado de: http:// www.jornada.unam.mx/20 I4/ I / /6/opinion/009a I pol

Sharaka (6 de febrero de 20I4). "Archivos". Sharaka. (perfil de comunidad en línea). Recuperado de: https://www. facebook.com/groups/360906 I77468/I 0 I5 I9582672। $7469 /$

Subcomandante insurgente Marcos (20 de noviembre de 1999). "Chiapas: la guerra. I. Entre el satélite y el microscopio, la mirada del otro". Discurso del subco- 
mandante Marcos ante la Comisión Civil Internacional de Observación de los Derechos Humanos. La Realidad, Chiapas. Recuperado de: http://enlacezapatista.ezln.org. $\mathrm{mx} /$ I 999/I I/20/chiapas-la-guerra-i-entre-el-satelite-yel-microscopio-la-mirada-del-otro-carta-5-I/

The Jerusalem Post (I7 de junio de 2014). "Hamas and ISIS". The Jerusalem Post. Recuperado de: http://www. jpost.com/Opinion/Editorials/Hamas-and-ISIS-359674

Thomas, B. (195I). "Migration and the rhythm of economic growth, I830-1913". Manchester School the Manchester School, 19(3), 215-27I.

Todorov, T. (2008). El miedo a los bárbaros más allá del choque de civilizaciones. Barcelona: Galaxia Gutenberg, Círculo de Lectores.

Vargas, L. (20I2). "El mito de la palabra a la pared. Murales zapatistas y el discurso chueco del subcomandante Marcos". Blanco Sobre Blanco. Miradas y Lecturas Sobre Artes Visuales, (2), 19-29. Recuperado de: https://www. academia.edu/5I0I I59/El_mito_de_la_palabra_a_la_ pared._Murales_zapatistas_y_el_discurso_chueco_del_ Subcomandante_Marcos

Vázquez, J. Z. (1977). Historia general de México 3. México, D.F.: El Colegio de México.

Zemelman, H. (2005). Voluntad de conocer: el sujeto y su pensamiento en el paradigma crítico. Barcelona/México: Anthropos/Centro de Investigaciones Humanísticas/ Universidad Autónoma de Chiapas.

Zibechi, R. (20I4). "La Escuelita zapatista: una nueva cultura política”. Desde Abajo. Revista de la Asociación Latinoamericana Para la Formación y la Enseñanza de la Psicología. Recuperado de: http://www.alfepsi.org/index.php/ reflexion-latinoamericana/3 /8-la-escuelita-zapatistauna-nueva-cultura-politica 